\title{
Transverse oscillation vector flow imaging for transthoracic echocardiography
}

\author{
Bradway, David; Lindskov Hansen, Kristoffer; Nielsen, Michael Bachmann; Jensen, Jørgen Arendt
}

\section{Published in:}

Proceedings of SPIE

Link to article, DOI:

$10.1117 / 12.2081145$

Publication date:

2015

Document Version

Publisher's PDF, also known as Version of record

Link back to DTU Orbit

Citation (APA):

Bradway, D., Lindskov Hansen, K., Nielsen, M. B., \& Jensen, J. A. (2015). Transverse oscillation vector flow imaging for transthoracic echocardiography. In J. G. Bosch, \& N. Duric (Eds.), Proceedings of SPIE (Vol. 9419). [941902] SPIE - International Society for Optical Engineering. Proceedings of SPIE - The International Society for Optical Engineering https://doi.org/10.1117/12.2081145

\section{General rights}

Copyright and moral rights for the publications made accessible in the public portal are retained by the authors and/or other copyright owners and it is a condition of accessing publications that users recognise and abide by the legal requirements associated with these rights.

- Users may download and print one copy of any publication from the public portal for the purpose of private study or research.

- You may not further distribute the material or use it for any profit-making activity or commercial gain

- You may freely distribute the URL identifying the publication in the public portal 


\title{
Transverse oscillation vector flow imaging for transthoracic echocardiography
}

\author{
David Pierson Bradway ${ }^{a}$, Kristoffer Lindskov Hansen $^{b}$, Michael Bachmann Nielsen ${ }^{b}$ and \\ Jørgen Arendt Jensen ${ }^{a}$ \\ ${ }^{a}$ Center for Fast Ultrasound Imaging, Department of Electrical Engineering, \\ Technical University of Denmark, DK-2800 Lyngby, Denmark; \\ ${ }^{b}$ Department of Radiology, Rigshospitalet, Copenhagen University Hospital, DK-2100 \\ Copenhagen, Denmark;
}

\begin{abstract}
This work presents the development and first results of in vivo transthoracic cardiac imaging using an implementation of Vector Flow Imaging (VFI) via the Transverse Oscillation (TO) method on a phased-array transducer. Optimal selection of the lateral wavelength of the transversely-oscillating receive field is described, and results from Field II simulations are presented. Measurements are made using the SARUS experimental ultrasound scanner driving an intercostal phased-array probe. The acquisition sequence was composed of interleaved frames of 68-line B-mode and 17-direction, 32-shot vector velocity flow images. A flow pump was programmed for constant flow for in vitro acquisitions at varying depths in a tissue-mimicking fluid. Additionally, mitral, aortic, and tricuspid valves of two healthy volunteers were scanned from intercostal acoustic windows. The acquired $\mathrm{RF}$ data were beamformed via the TO method, and fourth-order estimators were employed for the velocity estimation. The resulting images were compared with those from conventional spectral Doppler and color flow mapping sequences. VFI is shown to be a clinically-feasible tool, which enables new flexibility for choosing acoustic windows, visualizing turbulent flow patterns, and measuring velocities.
\end{abstract}

Keywords: Medical Ultrasound, Vector Flow Imaging, Cardiac Imaging, Blood Flow, Transverse Oscillation

\section{INTRODUCTION}

Velocity estimation of cardiovascular blood flow is an important clinical tool. Conventional color flow mapping (CFM) based on the autocorrelation approach ${ }^{1}$ allows the display of the 1-D axial velocities in a 2-D region of interest (ROI). This single component of velocity along the ultrasound beam does not provide a full representation of complex in vivo flow. With the introduction of $2-\mathrm{D}$ and $3-\mathrm{D}$ vector velocity algorithms, like the Transverse Oscillation (TO) method suggested by Jensen, Munk and Anderson, ${ }^{2,3}$ velocity direction and magnitude can be more accurately estimated without the need for angle correction or manual intervention.

A recent commercial implementation of the TO method on the ProFocus Ultra View scanner (BK Medical, Herlev, Denmark) runs in real-time for clinical vascular imaging on a linear array. Those tools have been utilized for studies measuring volume flow in arteriovenous fistulas ${ }^{4}$ and visualizing complex flow phenomena during intraoperative US examinations of the heart and aorta, ${ }^{5}$ where peak systolic velocity measurements were compared to conventional spectral Doppler velocities.

Expanding from the initial implementation on a linear array, recently a phased-array transducer was used for measurements in a flow rig, ${ }^{6}$ and a convex array was used for in vivo VFI and spectral measurements of flow in the vessels of the liver. ${ }^{7,8}$ This present work is the first known demonstration of in vivo cardiac VFI using the TO method from a noninvasive, transthoracic approach.

In this paper, the theory and optimization of TO fields are described in Section 2. Section 3 describes the methods and materials used for the development and pre-clinical testing. In Section 4, the flow phantom and in vivo results are presented and discussed, and Section 5 discusses the conclusions of this work.

Further author information: (Send correspondence to J. A. Jensen) E-mail: jaj@elektro.dtu.dk

Medical Imaging 2015: Ultrasonic Imaging and Tomography, edited by Johan G. Bosch,

Neb Duric, Proc. of SPIE Vol. 9419, 941902 - C 2015 SPIE

CCC code: $1605-7422 / 15 / \$ 18 \cdot$ doi: $10.1117 / 12.2081145$

Proc. of SPIE Vol. $9419941902-1$ 


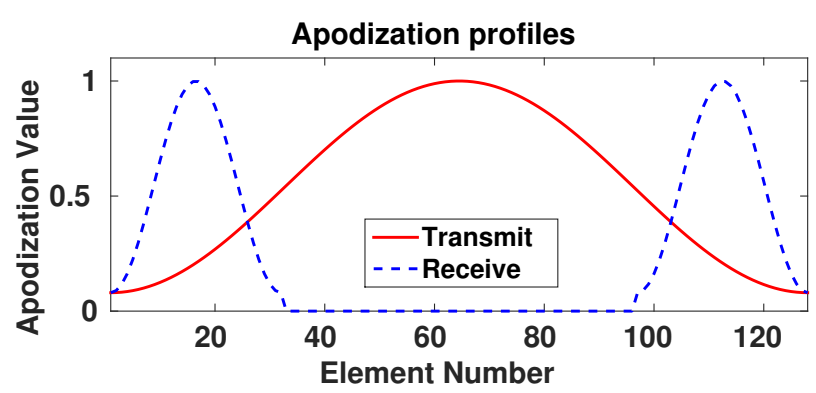

Figure 1: Transmit (red solid) and receive (blue dashed) apodization waveforms used during the simulation.

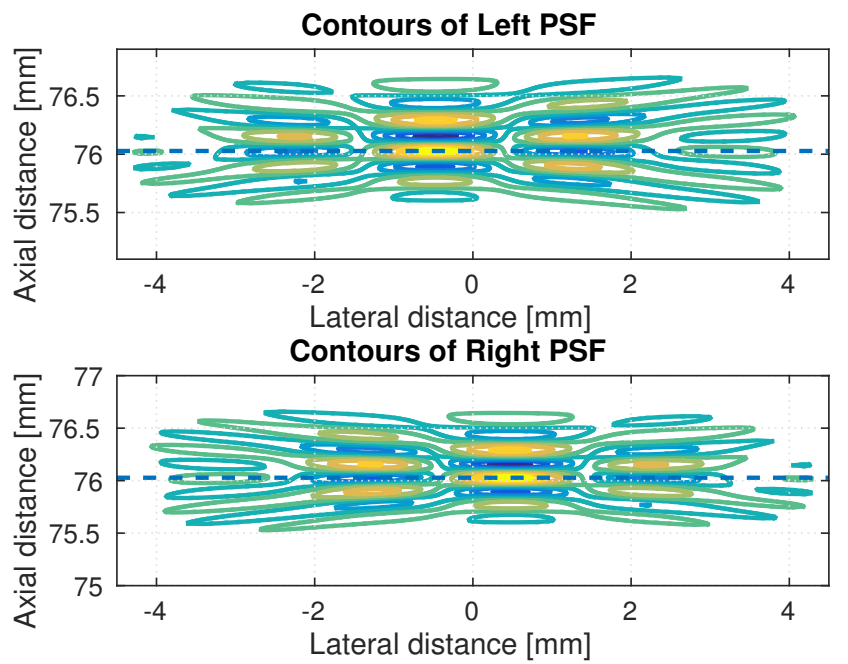

Figure 2: Linear contour plots of the left (top) and right (bottom) point spread functions at a depth of $75 \mathrm{~mm}$.

\section{THEORY AND OPTIMIZATION OF TO FIELDS}

For estimating velocity components transverse to the direction of propagation of the ultrasound beam, a weaklyfocused transmit field is repeatedly emitted at one or more positions in the ROI. A transversely-oscillating receive field can be constructed by applying apodization across the array composed of two Gaussian-weighted peaks, utilizing the Fourier relationship between the apodization function and the lateral response in the far field or at the focus. ${ }^{9}$ The receive apodization function is seen in Fig. 1 with two peaks separated by a distance $D$ generates a laterally-oscillating field at the focus with a wavelength $\lambda_{x}$ of ${ }^{2}$

$$
\lambda_{x}=2 \lambda_{z} \frac{z}{D},
$$

where $\lambda_{z}$ is the axial wavelength and $z$ the depth. The received lateral frequency is then

$$
f_{x}=v_{x} / \lambda_{x},
$$

where $v_{x}$ is the lateral component of the velocity.

Two beams are formed for each lateral position where velocity estimates are to be calculated. The linear contour plots of the left and right point spread functions are seen in Fig. 2. The fields are slightly displaced to either side of the center of the transmitted wave. The angle between focal directions for forming the left and right beams is adjusted so that the lateral Hilbert transform of the left beam is nearly equivalent to the lateral profile of the right beam, as seen in Fig. 3. The imperfect match between the profile of the right beam and the Hilbert transform of the left beam will cause leakage of energy into the negative frequencies in the 2-D Fourier transform 

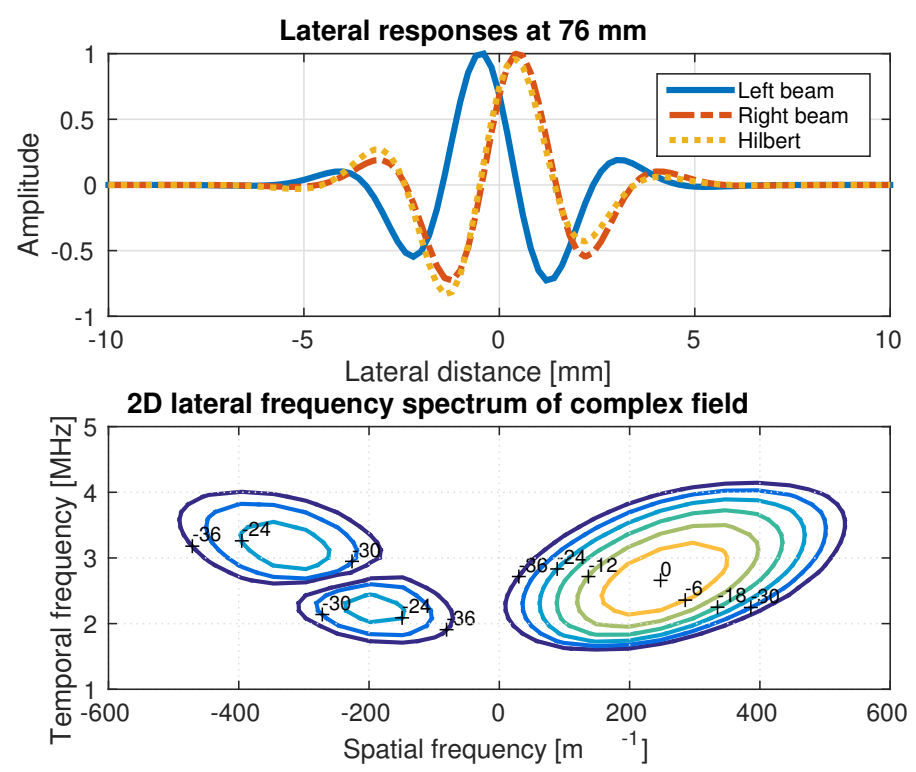

Figure 3: In the top plot, the lateral profiles of the PSFs are plotted for the left beam (solid blue), right beam (dashed red), and Hilbert transform of the left beam (dotted orange). The bottom plot shows the two-dimensional Fourier transform of the complex TO field.

Table 1: Parameters used for the optimization simulations.

\begin{tabular}{|l|c|}
\hline Transducer center frequency $f_{0}$ & $3.0 \mathrm{MHz}$ \\
Number of cycles in emitted pulse & 4 \\
Wavelength & $0.49 \mathrm{~mm}$ \\
Element pitch & $0.22 \mathrm{~mm}$ \\
Kerf & $22 \mu \mathrm{m}$ \\
Height of element & $15 \mathrm{~mm}$ \\
Fixed elevation focus & $85 \mathrm{~mm}$ \\
Number of physical elements of the transducer & 128 \\
Electronic focus in transmit & $113 \mathrm{~mm}$ \\
Depth for optimizing the transmit focusing & $75 \mathrm{~mm}$ \\
F\# in transmit for flow & 4 \\
Number of receive elements & $2 \times 32$ \\
PRF for flow & $6000 \mathrm{~Hz}$ \\
Emissions for flow & 32 \\
Directions for flow & 17 \\
\hline
\end{tabular}

of the complex TO field. This unwanted spectral leakage can be used as a criterion for the optimization routine. Samples from the left and right beams are used as in-phase and quadrature pairs in the lateral direction.

The TO field was optimized for imaging at $75 \mathrm{~mm}$ by adjusting the beamforming and receive apodization function using the approach described by Jensen. ${ }^{10}$ The transducer specifications and imaging parameters used in the optimization study are shown in Table 1.

In Fig. 4, the desired oscillation period is plotted against the achieved simulated lateral wavelength. Setting the lateral wavelength to $4 \mathrm{~mm}$ to determine for the receive beams' focus will result in a match between desired and actual wavelength for this focal position. 


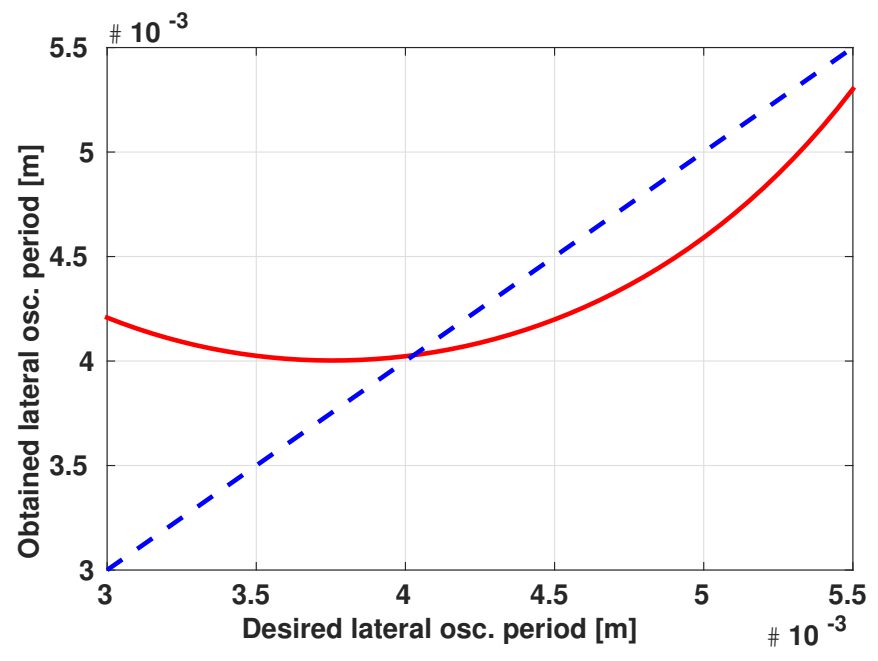

Figure 4: This plots the obtained lateral oscillation period in solid blue as a function of the desired lateral oscillation period in dashed red. Near $4 \mathrm{~mm}$ the lines cross, indicating good agreement between desired and achieved lateral period.

This approach is applied to a phased array receive field, and vector flow data are acquired in a flow phantom and in vivo as described in the next section.

\section{EXPERIMENTAL SETUP}

The SARUS experimental ultrasound scanner has been used for acquiring data ${ }^{11}$ utilizing a $3 \mathrm{MHz}$ STV4 phased-array probe with 128 elements (Sound Technology, Analogic Ultrasound Group, State College, PA). The acquisition sequence consisted of alternating frames of a 68 -line B-mode image and a 17-direction, $N_{l}=32$ flow shot VFI ensemble. The transmit focus was $56 \mathrm{~mm}$ for B-mode and $113 \mathrm{~mm}$ for VFI.

\subsection{In vitro}

Preliminary experimental measurements were conducted on a flow phantom containing a $3.6 \mathrm{~mm}$ radius tube embedded $10 \mathrm{~mm}$ within a tissue-mimicking material. Tissue-mimicking fluid was added atop the phantom and the scanning depth was varied from $45 \mathrm{~mm}$ to $85 \mathrm{~mm}$.

\subsection{In vivo}

To ensure FDA compliance for in vivo scanning, hydrophone intensity measurements were acquired with an AIMS III scanning system and software (Onda, Sunnyvale, CA). An experienced radiologist acquired in vivo measurements of two healthy volunteers' mitral, aortic, and tricuspid valves. A ProFocus scanner was used for ROI guidance and comparison measurements and images. Cine loops and still images were saved for B-Mode, CFM, and spectral Doppler imaging sequences.

\subsection{Data processing}

Data from the SARUS research scanner were sampled for off-line processing in MATLAB (The MathWorks, Inc., Natick, MA, USA) under Linux. The stored raw RF data were matched-filtered and beamformed using the Beamformation Toolbox $3 .{ }^{12}$ Mean stationary echo canceling (clutter filtering) was performed by subtracting the mean value of $N_{l}$ ensemble lines before velocity estimation. Algorithms outlined in previous work ${ }^{6}$ were used for estimation of axial and transverse flow directions. Estimated velocity components were rotated by the steering angle and were scan converted to a Cartesian grid using bilinear interpolation.

ROIs were marked in the B-Mode by a radiologist, and the corresponding flow estimates were extracted. The magnitude of the flow was found for each point in the ROI, and the mean and standard deviation were calculated. 


\section{RESULTS AND DISCUSSION}

\subsection{In vitro}

Results from the deepest flow phantom acquisition are shown in Fig. 5. The flow within the vessel is masked with a marked ROI. The measured velocity had mean velocity $20.8 \pm 8.8 \mathrm{~cm} / \mathrm{s}$. The angle of flow in the ROI had a mean angle of $3.3 \pm 10.2$ degrees. There was some uncertainty in alignment of the transducer fixture with the angle of flow in the rig, but qualitatively there was good agreement with the estimated direction.

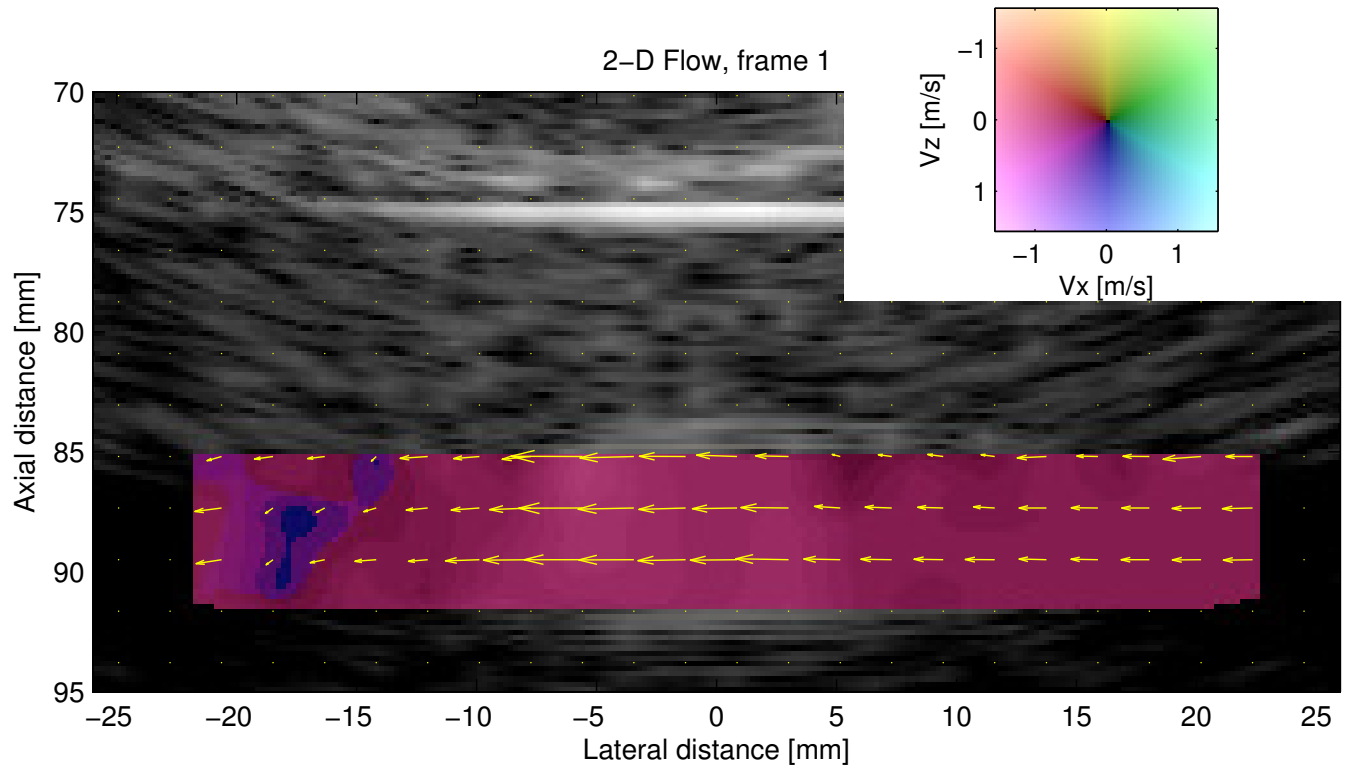

Figure 5: 2-D velocity vectors in a flow rig at $85-92 \mathrm{~mm}$ depth, with arrows that indicate the velocity direction and relative magnitude with corresponding 2-D color wheel map

\subsection{In vivo}

The derated intensity values were $\mathrm{MI}=0.8$ and $I_{\text {spta. } 3}=66 \mathrm{~mW} / \mathrm{cm}^{2}$. Thirty measurements from an in vivo VFI acquisition from the second volunteer's tricuspid valve are shown in Fig. 6 . The dataset was obtained from right parasternal long axis view. The shape and pattern of the magnitude of the VFI-derived velocity profile appears comparable to the absolute value of the angle-corrected estimates obtained conventional spectral Doppler imaging using the ProFocus scanner, with peak velocity magnitude around $100 \mathrm{~cm} / \mathrm{s}$.
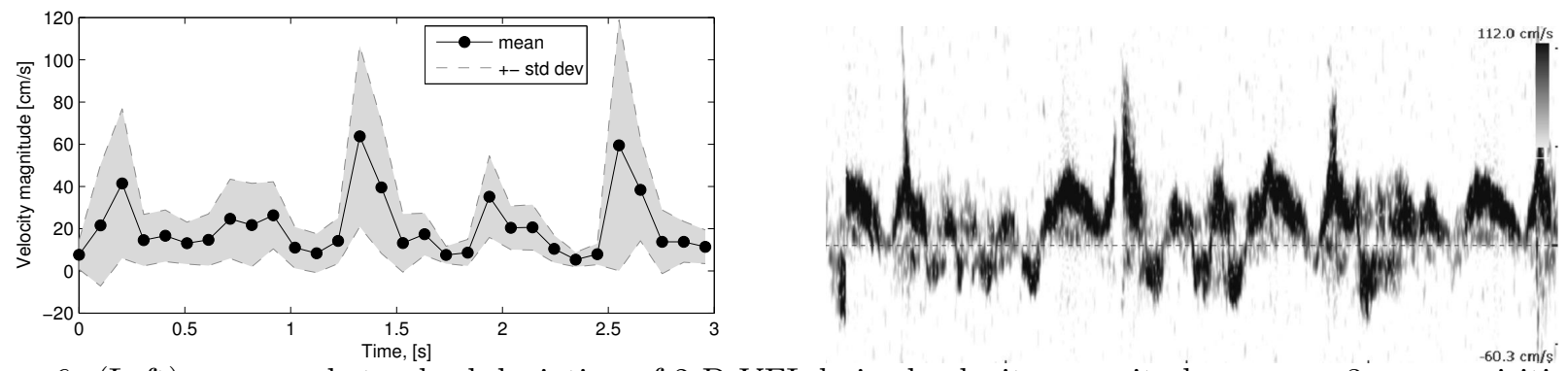

Figure 6: (Left) mean and standard deviation of 2-D VFI-derived velocity magnitude across a 3-sec acquisition and (Right) the corresponding 1-D angle-corrected velocity estimates from conventional spectral Doppler imaging, where velocities range from $-60.3 \mathrm{~cm} / \mathrm{s}$ to $+112.0 \mathrm{~cm} / \mathrm{s}$ and the acquisitions cover nearly 4 seconds.

One image frame from this tricuspid acquisition and another frame from the same volunteer's aortic valve are shown in Fig. 7. The direction and magnitude velocity estimates can be seen in the ROI. This view provides new information into complexity of the flow patterns, which may be useful for judging valve health and turbulence in the sinuses. 

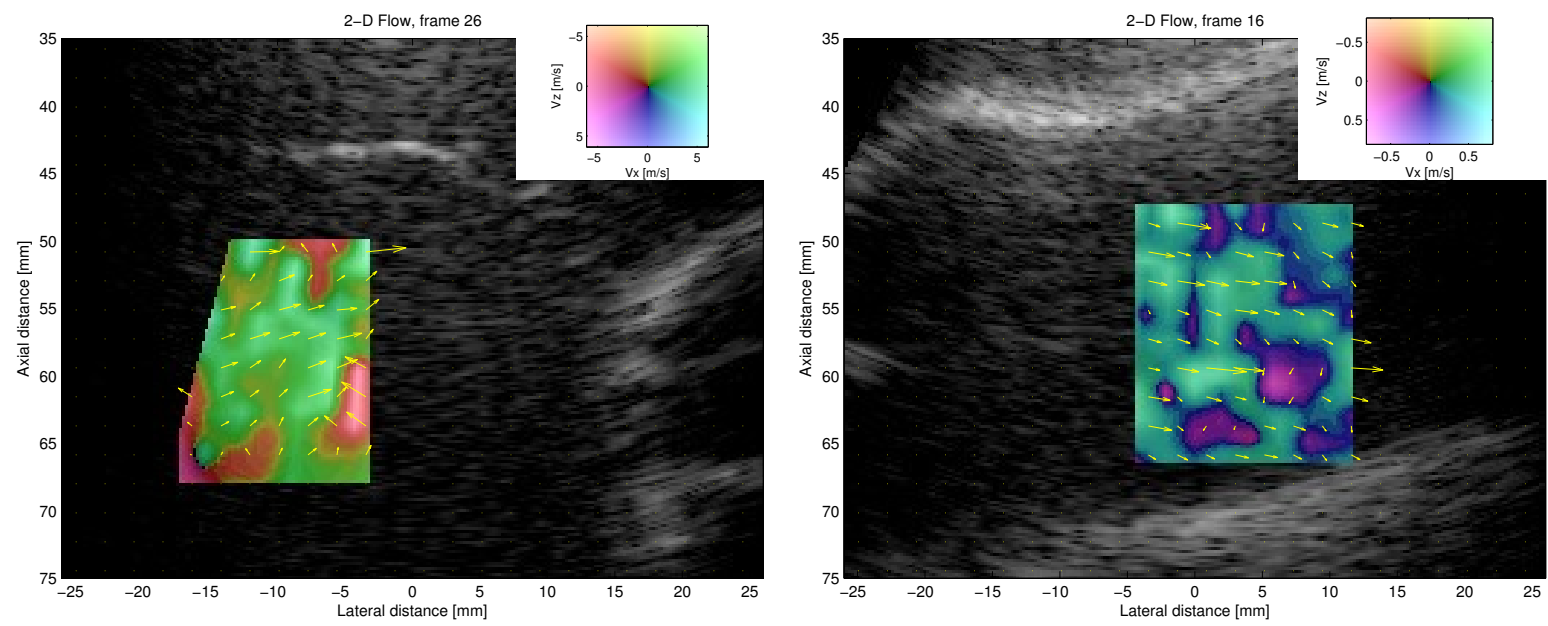

Figure 7: B-Mode image frames with VFI colors and velocity arrows overlayed inside the ROI. (Left) contains a frame from the tricupsid valve acquisition and (Right) shows flow in the aorta, with the aortic valve at the left edge of the image.

In Fig. 8, a conventional color flow mode (CFM) image is shown. Since the main flow is nearly perpendicular to the ultrasound propagation direction, this can be problematic for the precise measurement of flow velocities and interpretation of the CFM image from this acoustic window. The conventional color map switches abruptly from red to blue as the flow direction changes with respect to the steering angle of the transmitted pulses. Trained cardiologists and sonographers are familiar with reading the conventional CFM image color map, but the 2-D color wheel map of VFI provides more information about the true magnitude and direction of complex cardiac flow without relying on operator intervention for angle correction.

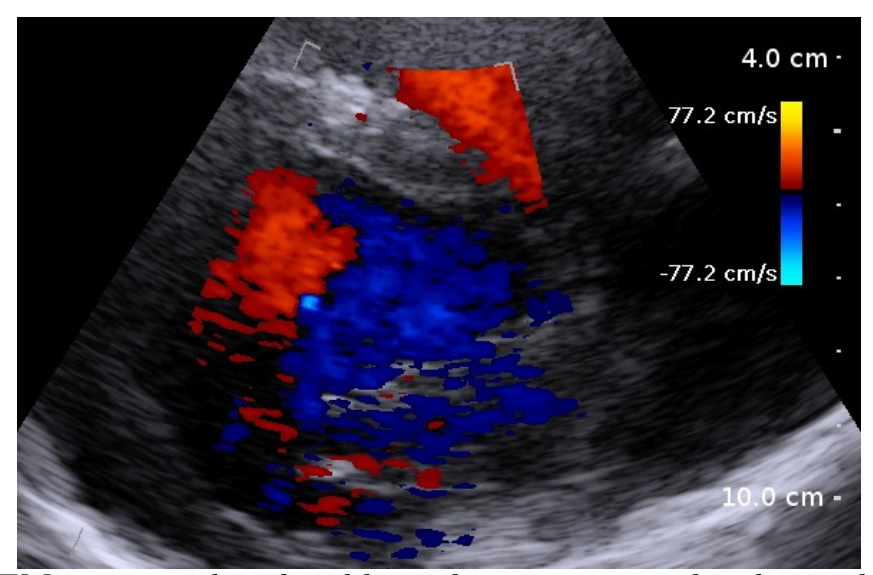

Figure 8: Conventional CFM image with red to blue color mapping scale, obtained from a ProFocus scanner.

\section{CONCLUSION AND PERSPECTIVES}

This work demonstrated the development of VFI on a phased array. First, optimization simulations were completed and flow measurements made at depths up to $9 \mathrm{~cm}$ in a programmable flow rig. Transthoracic in vivo cardiac scans were performed for the tricuspid, mitral and aortic valves. Results were compared to conventional spectral Doppler velocity measurements and CFM images. 
This initial study had some limitations, including sample size, and a larger pre-clinical study has been completed which will address repeatability and variability. Processing and display were performed off-line in this work, but the TO method and estimation algorithms are suitable for real-time use, as is presently done in the BK Medical linear array VFI mode or by using a graphics processing unit (GPU) in an attached research workstation as shown at this meeting last year. ${ }^{13}$ The frame rate achieved in this work was insufficient to sample the temporal dynamics of the heart around peak systole, so in future work, synthetic aperture flow should be pursued to enable continuous sampling and estimation at a higher frame rate.

As a diagnostic tool, VFI will improve guidance and feedback to cardiologists and researchers through improved visualization of turbulence and the removal of the angle-correction of conventional spectral Doppler estimation.

\section{ACKNOWLEDGMENTS}

The author thanks Borislav Tomov and Matthias Bo Stuart for technical support. Additionally, this work was supported by grant 82-2012-4 from the Danish Advanced Technology Foundation, by BK Medical, and by a

postdoctoral scholarship from the Whitaker International Program, administered by the Institute of International Education (New York, NY, USA).

\section{REFERENCES}

[1] Kasai, C., Namekawa, K., Koyano, A., and Omoto, R., "Real-Time Two-Dimensional Blood Flow Imaging using an Autocorrelation Technique," IEEE Trans. Son. Ultrason. 32, 458-463 (1985).

[2] Jensen, J. A. and Munk, P., "A new method for estimation of velocity vectors," IEEE Trans. Ultrason., Ferroelec., Freq. Contr. 45, 837-851 (1998).

[3] Anderson, M. E., "Multi-dimensional velocity estimation with ultrasound using spatial quadrature," IEEE Trans. Ultrason., Ferroelec., Freq. Contr. 45, 852-861 (1998).

[4] Hansen, P. M., Olesen, J. B., Pihl, M. J., Lange, T., Heerwagen, S., Pedersen, M. M., Rix, M., Lønn, L., Jensen, J. A., and Nielsen, M. B., "Volume flow in arteriovenous fistulas using vector velocity ultrasound," Ultrasound Med. Biol. 40(11), 2707-2714 (2014).

[5] Hansen, K. L., Pedersen, M. M., Møller-Sørensen, H., Kjaergaard, J., Nilsson, J. C., Lund, J. T., Jensen, J. A., and Nielsen, M. B., "Intraoperative cardiac ultrasound examination using vector flow imaging," Ultrason. Imaging 35, 318-332 (Oct 2013).

[6] Pihl, M. J., Marcher, J., and Jensen, J. A., "Phased-array vector velocity estimation using transverse oscillations," IEEE Trans. Ultrason., Ferroelec., Freq. Contr. 59(12), 2662-2675 (2012).

[7] Jensen, J. A., Brandt, A. H., and Nielsen, M. B., "In-vivo convex array vector flow imaging," in [Proc. IEEE Ultrason. Symp.], 333-336, IEEE (2014).

[8] Jensen, J. A., "Transverse spectral velocity estimation," IEEE Trans. Ultrason., Ferroelec., Freq. Contr. 61, 1815-1823 (November 2014).

[9] Goodman, J. W., [Introduction to Fourier optics], McGraw Hill Inc., New York, second ed. (1996).

[10] Jensen, J. A., "Optimization of transverse oscillating fields for vector velocity estimation with convex arrays," in [Proc. IEEE Ultrason. Symp.], 1753-1756 (July 2013).

[11] Jensen, J. A., Holten-Lund, H., Nilsson, R. T., Hansen, M., Larsen, U. D., Domsten, R. P., Tomov, B. G., Stuart, M. B., Nikolov, S. I., Pihl, M. J., Du, Y., Rasmussen, J. H., and Rasmussen, M. F., "SARUS: A synthetic aperture real-time ultrasound system," IEEE Trans. Ultrason., Ferroelec., Freq. Contr. 60(9), 1838-1852 (2013).

[12] Hansen, J. M., Hemmsen, M. C., and Jensen, J. A., "An object-oriented multi-threaded software beamformation toolbox," in [Proc. SPIE Med. Imag.], 7968, 79680Y 1-9 (March 2011).

[13] Bradway, D. P., Pihl, M. J., Krebs, A., Tomov, B. G., Kjær, C. S., Nikolov, S. I., and Jensen, J. A., "Real-time gpu implementation of transverse oscillation vector velocity flow imaging," in [SPIE Medical Imaging], 90401Y-90401Y, International Society for Optics and Photonics (2014). 